\title{
GRAPHITE AS A PLASMA-FACING MATERIAL IN FUSION EXPERIMENTS*
}

\author{
R. A. Langley \\ Oak Ridge National Laboratory, P.O. Box 2009, Oak Ridge, TN 37831-8072
}

\section{ABSTRACT}

Graphite is now used extensively in most of the major fusion experiments in the world and will be used more extensively in future devices. In addition to its excellent tolerance of high heat fluxes, graphite has many unusual characteristics that pertain to its use as a plasma-facing material; these are its propensity for releasing gases when heated and when exposed to ion fluxes, its ability to absorb copious quantities of hydrogen during hydrogen bombardment, and its ability to pump hydiogen after noble gas bombardment.

The graphite used in existing machines and considered for use in future machines is isotropic on a macroscopic scale and anisotropic on a microscopic scale; it has a large open porosity, up to $20 \%$. This leads to enormous internal surface areas for adsorption and desorption of gases. Most early hydrogen-graphite interaction experiments were incorrectly analyzed because of this property. In addition, interaction of energetic hydrogen ions with graphite can lead to erosion, with concomitant deposition of carbon films with high hydrogen content on chamber surfaces. These effects are observed experimentally and have been modeled with some success.

This paper presents experimental data dealing with these topics and their influences or present-day plasma operations and on graphite use in future machines.

\section{INTRODUCTION}

Graphite is considered one of the few materials suitable for plasma-facing components in magnetic fusion experiments. Its main advantages are its excellent hightemperature properties and its low atoric number $(Z=6)$. Its major disadvantages are its propensity for releasing gases when heated and when exposed to ion fluxes, its ability to absorb copious quantities of hydrogen during energetic hydrogen bombardment, and its erosion when exposed to hydrogen bombaráment, especially at higher temperatures.
Among the outstanding general characteristics of graphite that make it suitable for use in fusion experimen's are the following.

1. It becomes stronger as its temperature increases; its strength is unexcelled at very high remperatures.

2 It has thermal and electrical characteristics usually associated with metals.

3. It has excellent thermal shock resistance for a brittle material.

4. It is highly inert to chemical attack at low to moderate temperatures.

In addition, graphite has many characteristics that can vary over a wide range. Table I sho'vs the limits of variability for some of the more important characteristics pertinent to its use in fusion experiments. In general, the more isotopic the graphite, the better suited it is to structural, thermal, and electrical applications in fusion experiments. High isotropy dictates small grain size and generally leads to relatively large open porosity. To limit the absorbed gas and hydrogen uptake, very low porosity and internal surface area are desired. However, highly isotropic material has most often been selected because it is available in large quantities and different forms at relatively low cost and is easily machined.

Table I. Pertinent graphite characteristics and limits of variability

\begin{tabular}{lll}
\hline & Natural/pyrolytic & Artificial \\
\hline Isotropy & Anisotropic & Isotropic \\
Crystal size & $1 \mathrm{~cm}$ & $1-500 \mu \mathrm{m}$ \\
Density & $2.26 \mathrm{~g} / \mathrm{cm}^{3}$ & $1.6 \mathrm{~g} / \mathrm{cm}^{3}$ \\
& (theoretical) & \\
Porosity & $-0 \%$ & $20 \%$ \\
Connected internal & -0 & $0.1-1 \mathrm{~m}^{2} / \mathrm{g}$ \\
surface area & & \\
\hline
\end{tabular}

\footnotetext{
"Research sponsored by the Office of Fusion Energy, U.S. Deparment of Energy, under contract DE-AC05-84OR21400 wi!'، Martin Marietta Energy Systems, Inc.
} 
This choice has led to problems in the areas of absorbed gas removal and hydrogen retention, especially with regard to tritium retention.

This paper addresses mainly the areas of hydrogen retention and release, which are important in the operation and safety of fusion experiments. Section II presents some background material on graphite that is basic to the following discussions. Section III addresses the results of experiments that deal with the near-surface region, defined as the region between the surface and the end of the range for energetic hydrogen implantation. Section IV addresses the pore surface, and Sec. $V$ discusses intragranular phenomena. Section VI presents the results of experiments in which hydiogen retention has been observed in sputtered carbon films deposited on the internal surfaces of experiments. Section VII discusses the questions that remain to be answered.

\section{BACKGROUND}

Since many of the experimental results are dependent on the type of graphite used in an experiment, a brief discussion of the various types of graphite is in order. Graphite is made up of carbon atoms arranged in a hexagonal pattern (see Fig. 1). The iayers of carbon atoms (i.e., the basal planes) are

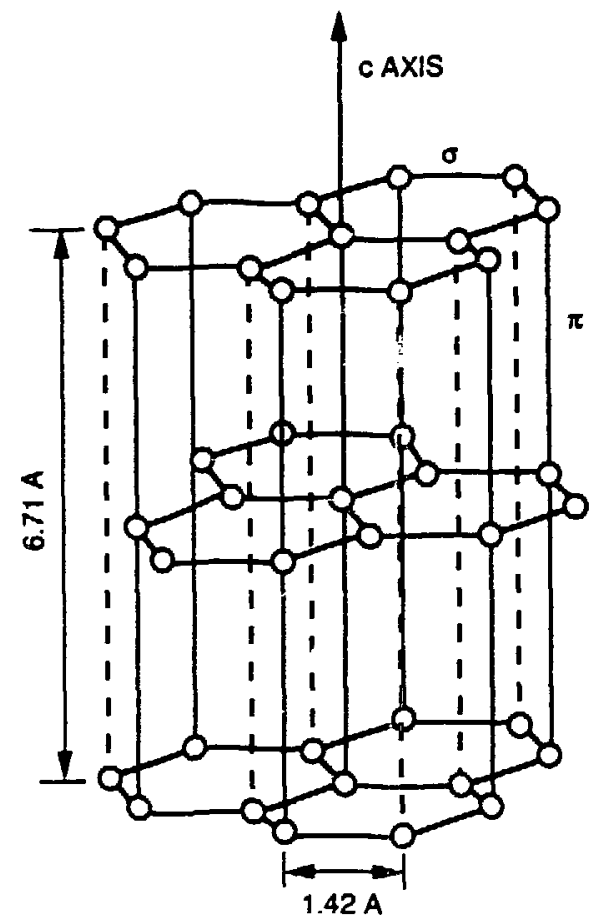

Fig. 1. Schematic of graphite structure. stacked with an $a b a b$ scheme. Each carbon atom has four valence electrons: three $\sigma$ electrons and one $\pi$ electron. The $\sigma$ electrons form strong covalent bonds with their nearest neighbors in the plane, and the $\pi$ electron is loosely bound [1]. The planes are separated by $3.35 \AA$, and their nearestneighbor spacing is $1.42 \AA$. Each carbon atom on the end of a plane has only two nearest-neighbor carbon atoms, so a $\sigma$ electron is available for bonding to atoms or moleciles that come into cuitact with this "active" carbon atom. For the lattice parameters given here, the number of active carbon atoms per unit area perpendicular to the basal plane is $1.3 \times$ $10^{19} \mathrm{~m}^{-2}$. Based on the strength of the $\mathrm{C}-\mathrm{H}$ bond in benzene, a reasonable estimate for the trapping energy of hydrogen on the active carbon atom is $4.3 \mathrm{eV}$ [2].

In natural graphite, single crystals as large as a few centimeters have been found. Pyrolytic graphite can be produced by the pyrolysis of carbonaceous vapors (e.g., $\mathrm{CH}_{4}$ ) on a suitable substrate at temperatures near $2000^{\circ} \mathrm{C}$. This results in layers with near-perfect alignment of the basal planes to the c-axis, but with considerable misalignment perpendicular to the c-axis. Crystals of this type cas be quite large (a few centimeters). In general, both natural and pyrolytic graphite are highly anisotropic, have densities near the theoretical density of $2.26 \mathrm{~g} / \mathrm{cm}^{3}$, and have very low porosity. In contrast, artificial graphite has crystallite sizes that can vary from one to a few hundred micrometers. These crystallites are formed into grains that are randomly oriented and connected by petroleum pitch that has been graphitized, that is, annealed at temperatures above $1700^{\circ} \mathrm{C}$ [3]. This leads to isotropy on a macroscopic scale and a large open porosity.

In an early experimental study [4], Montet documenied qualitatively many of the phenomena studied quantitatively in recent years: a large amount of hydrogen (much greater than the amount taken up in the near-surface region) could be taken up by graphite under hydrogen bombardment; the amount taken up decreased with increasing temperature; sputtered carbon films created during hydrogen bombardment took up a considerable amount of hydrogen; annealing the graphite to $1000^{\circ} \mathrm{C}$ would thermally release most of the implanted hydrogen; and almost no helium was taken up when a discharge was run with helium. All of these findings have now been quantified and to a large extent explained by using models that fit the experimentai data.

\section{NEAR-SURFACE}

The urapping and release of hydrogen from the nearsurface region of graphite have been studied extensively and 
are understood to a large degree, although some questions remain [5]. The near-surface region is defined as the region between the surface and the end of the range of the implanted ions. Graphite has been observed to retain all nonreflected hydrogen until a saturation concentration is reached in the implantation region; for room temperature, this concentration is $-0.5 \mathrm{H} / \mathrm{C}$, as demonstrated by Langley et al. [6] for 8-keV $D^{+}$implantation into pyrolytic graphite at room temperature (see Fig. 2) and verified by other experimenters for other types of graphite [7,8]. The implantation profile was initially observed to show a typical range-energy profile, but with increasing fluence the profiles spread toward the surface with increasing fluence once saturation was reached. This effect has been shown to be due to implant damage trapping [9-11]. The damage due to implantation begins as twinning and proceeds to amorphization of the implant region. The critical fluencu for amorphization is dependent on the incident ion, its energy. and the substrate temperature. For incident deuterium and helium ions, the transition from crystalline to amorphous varies with implantation temperature and with displacements per atom (dpa) [9] such that as the temperature increases the dpa level required for transition from crystalline to amorphous increases; ai $100^{\circ} \mathrm{C}$ only about $1 \mathrm{dpa}$ is needed, but at $500^{\circ} \mathrm{C}$ about $10 \mathrm{dpa}$ is needed (see Fig. 3). The total amount of hydrogen retained at samuration depends directly on the range of the implanted

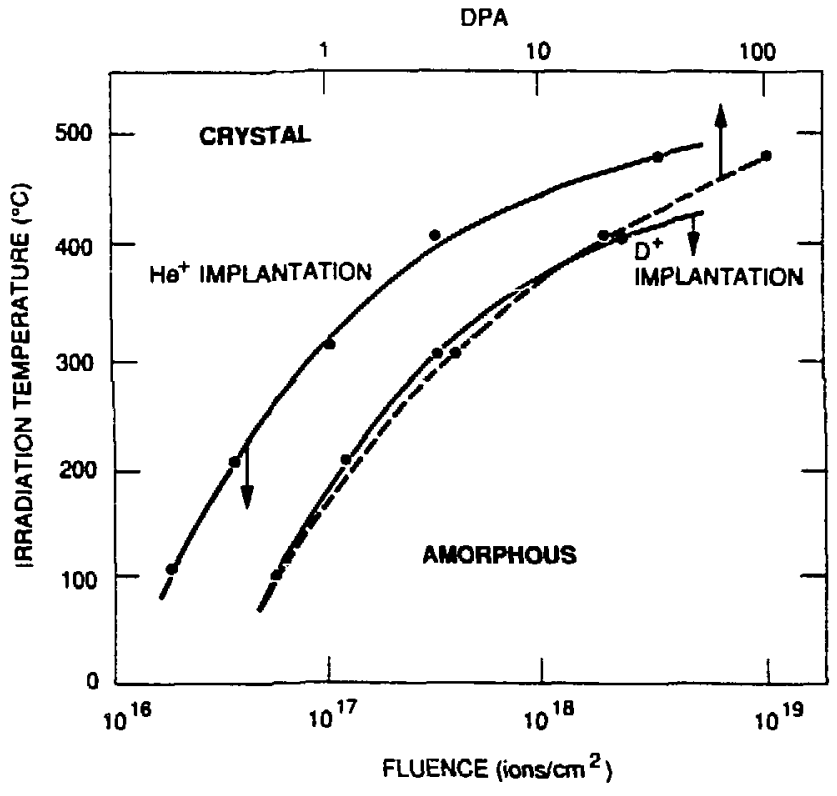

Fig. 3. Critical fluence and dpa levels for transition from crystal to amorphous vs irradiation temperature (from Ref. (9]).

ions and on the temperature of the substrate during implantation. The calculated ranges of energetic hydrogen, deuterium, and helium are shown in Fig. 4 as functions of energy for energies to $2 \mathrm{keV}$ [12]. The profiles are rather

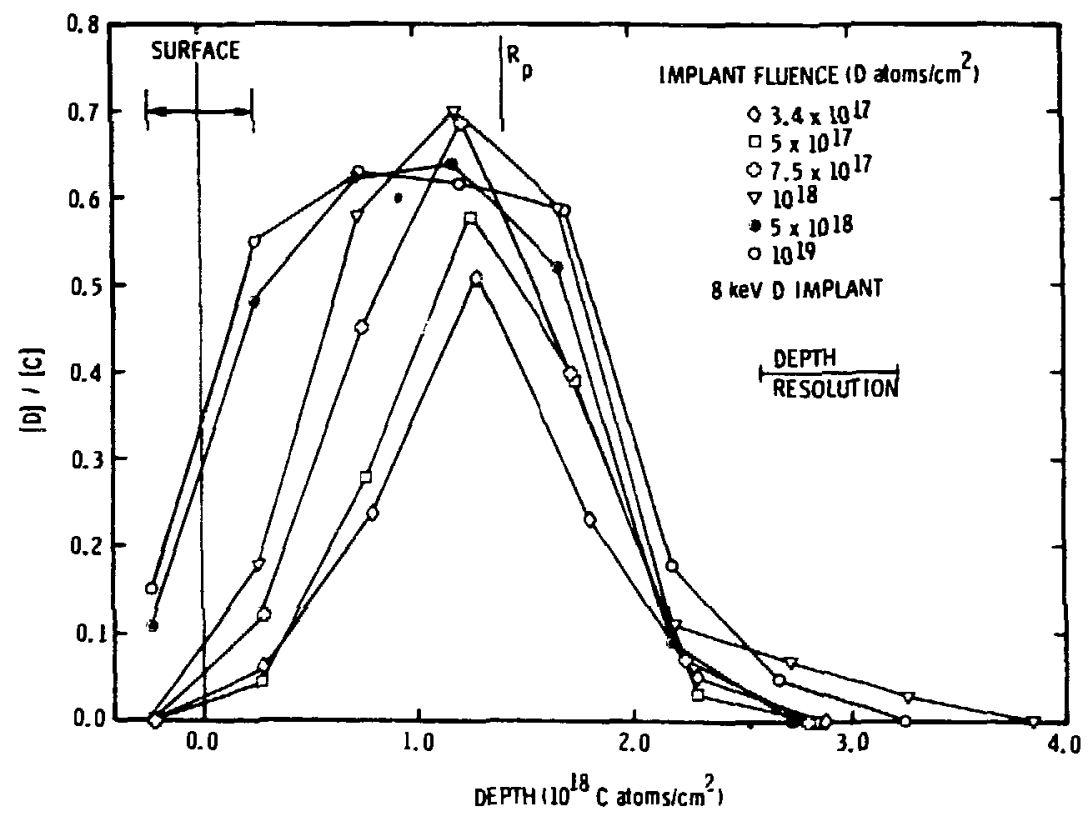

Fig. 2. Depth profiles for implanted 8-keV deuterium as a function of implant fluence at room temperature (from Ref. [6]). 


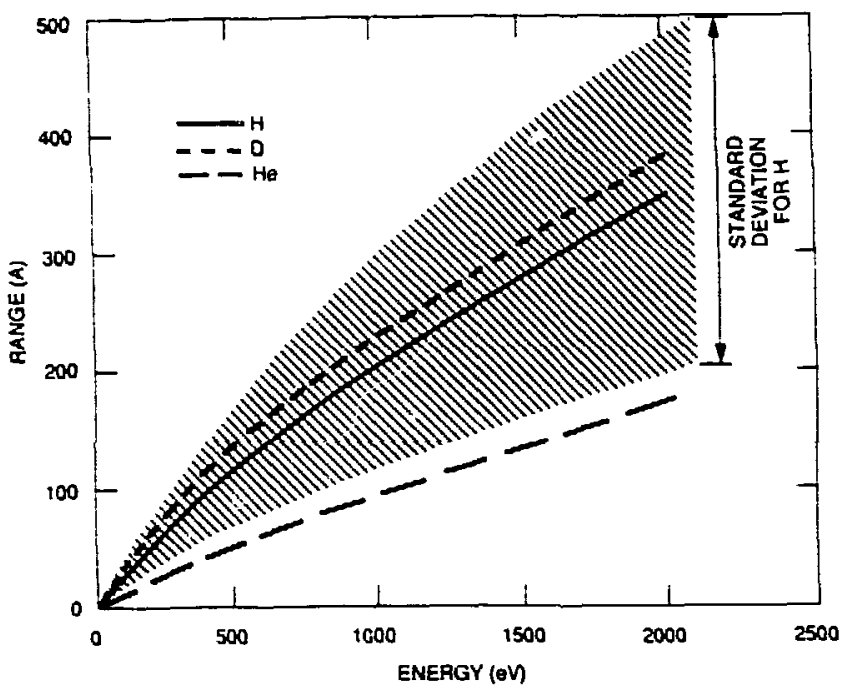

Fig. 4. Calculated projected range curves for $H, D$, and He in carbon. Also shown is the mean square deviation of the range for hydrogen to illustrate the profile width (from Ref. [12]).

broad at these energies; the mean square deviation of the range is shown for hydrogen as an example. The absolute amount of hydrogen retained as a function of fluence for implant energies to $5 \mathrm{keV}$ is shown in Fig. 5 for roomtemperature implants $[7,8,13]$. The $100 \%$ retention line is shown for reference. At low energies ( $<300 \mathrm{eV})$, the reflection coefficient is quite large, as is seen for the $E=$

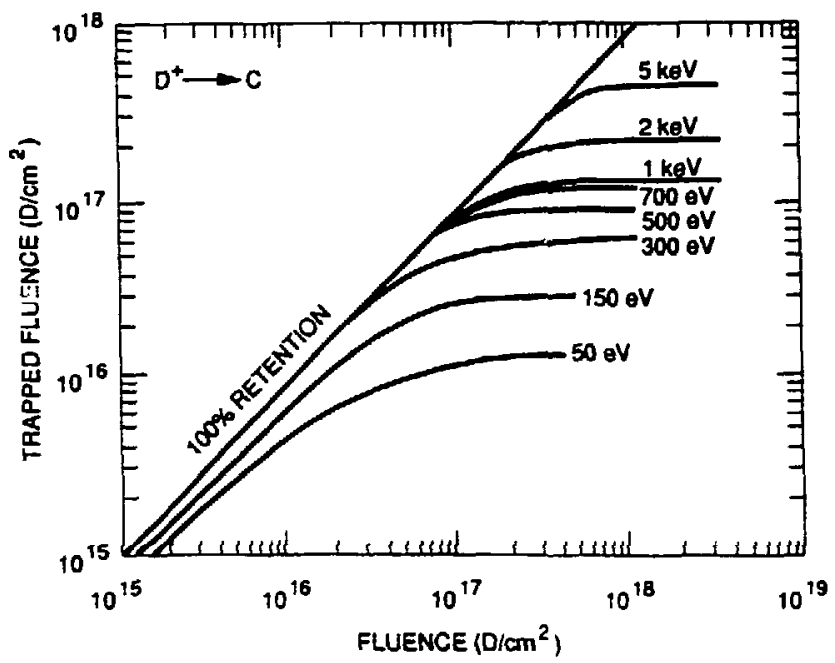

Fig. 5. Absolute amount of hydrogen retained as a function of fluence for room-temperature implants. The $100 \%$ retention line is shown for reference (from Refs. $[7,8,13]$ ).
$50 \mathrm{eV}$ and $150-\mathrm{eV}$ curves, where the retention curves are lower than the $100 \%$ retention curve even for the lowest fluence. Saturation of the near-surface region occurs at a hydrogen fluence of about $2 \times 10^{17} \mathrm{~cm}^{-2}$ for $50-\mathrm{eV}$ ions and increases with increasing energy. At implantation temperatures above $150^{\circ} \mathrm{C}$, the saturation level decreases with increasing temperature; see Fig. 6 [13]. This can be atributed to the effects of themally influenced detrapping and recombination $[13,14]$. Since retention and release have been found to be dependent on thermal detrapping, ioninduced detrapping, temperature-dependent recombination, and inward pore-surface migration, it is not surprising that earlier experimental results were not thought to be consistent and were experiment dependent. Figure 7 shows the effects of postimplantation annealing on the retention for three experiments at different energies $[6,8,13]$. Recently, Brice [14] has made significant advances toward resolving earlier inconsistencies by including temperature-dependent recombination in modeling the processes discussed above.

Ion-induced release of hydrogen from the hydrogensaturated near-surface region has been measured for hydrogen, deuterium, helium, and carbon ions at room temperature [15-19]. The incident energies range from 100 $\mathrm{eV}$ to $2.5 \mathrm{MeV}$. Additional data are needed at higher temperatures. The experimental results obtained have been successfully modeled by including detrapping. recombination, and migration on grain surfaces. The nearsurface region from which hydrogen is removed remains in the amorphous state and is readily available for future

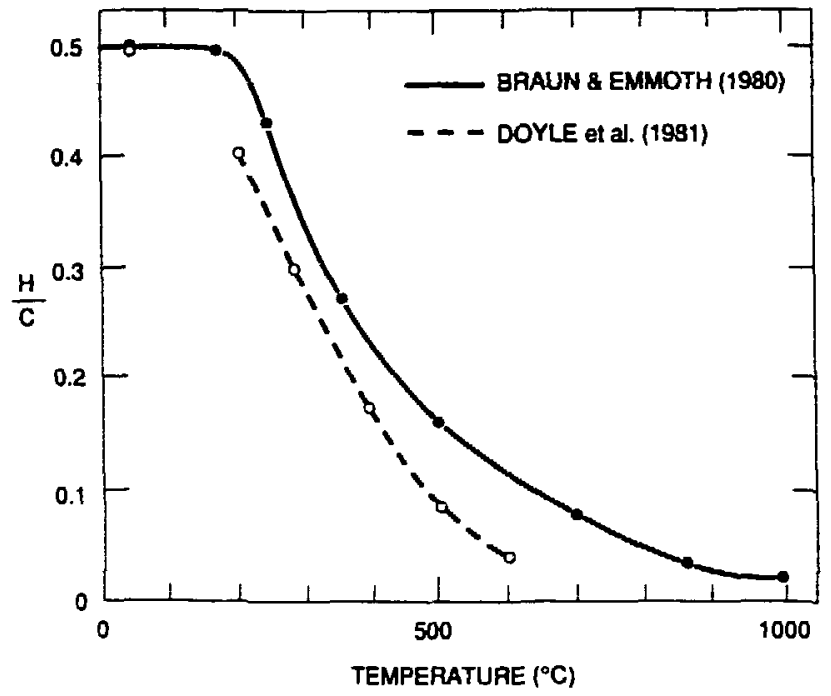

Fig. 6. Saturation level of hydrogen in the near-surface region of graphite as a function of implant temperature (from Ref. [13]). 


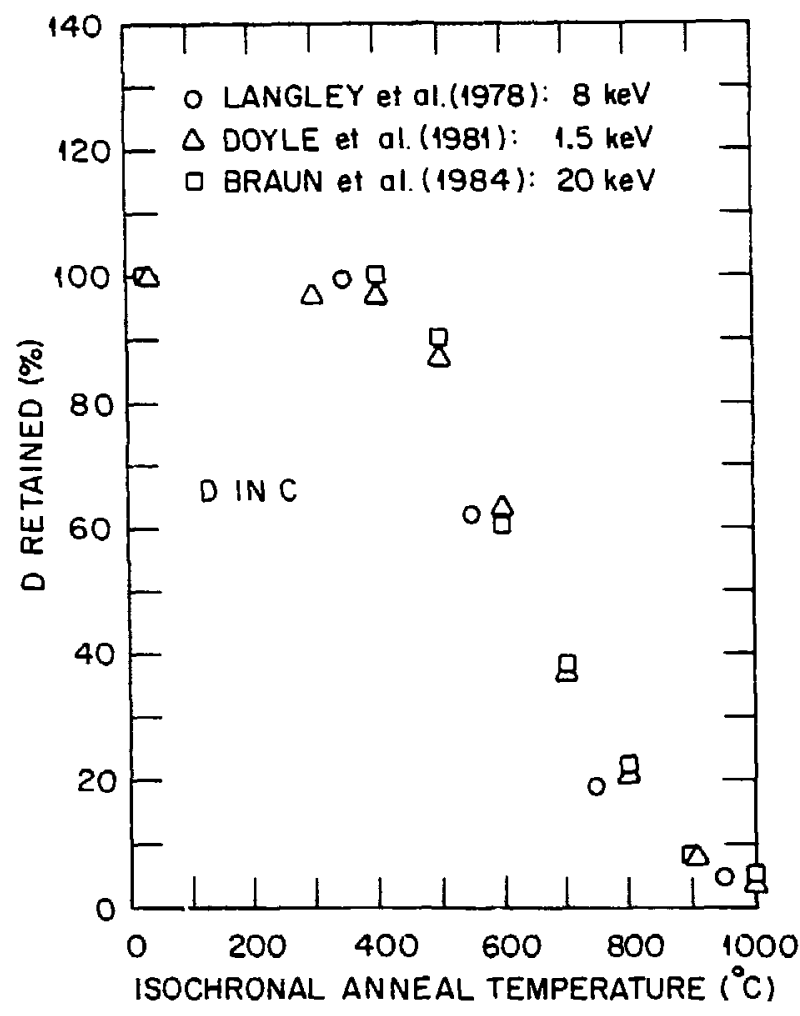

Fig. 7. Implanted deuterium retained in near-surface region vs isochronal anneal temperature.

retention of hydrogen, as observed in both laboratory experiments [18,19] and tokamak experiments $[20,21]$.

The replacement of hydrogen and deuterium in the nearsurface region has been studied $[8,15,19]$ and successfully modeled with the local mixing model [20]. Detrapping is caused by the incident particles, and there is competition for the vacant trap by detrapped particles and implanted particles. It appears quite feasible to replace one hydrogen isotope with another in the near-surface region using a glow discharge, and this process could be used to reduce the tritium inventory in the near-surface region $[8,19]$.

\section{PORE SURFACE}

The retention of hydrogen in graphite has been studied extensively. Many of the results appear contradictory. The observed differences have been variously attributed to the state in which the hydrogen was introduced (atomic or molecular, neutral or ionized), to the grade of graphite used, and to the hydrogen detection $t$-hnique. In addition, significant differences have been observed for the temperature dependence of the retention.
In 1959, Montet [4] reported significant retention of hydrogen in graphite-much more than could be accounted for by that retained in the near-surface region. Langley [22] quantified the amount retained as a function of incident fluence and flux over wide ranges and as a function of incident energy from, 125 to $250 \mathrm{eV}$ for room-temperature implantation and for artificial graphite (AXF-5Q). The percentage of hydrogen retained was found to remain constant with increasing fluence but to decrease with increasing flux, see Fig. 8.

Recent unpublished results by Haasz [23] show similar results for the fluence dependence down to temperatures of $125^{\circ} \mathrm{C}$. In contrast, Causey et al. [24] and Hsu and Causey [25] obtained much lower values for retention following a single high-flux implantation. Causey and Hsu found retention of only $-0.1 \%$ near room temperature for a flux about 50 times the maximum used by Langley. Since Langley found that retention decreased with increasing flux, these results may not actually be mutually exclusive. According to Causey et al. [24], at temperatures between $23^{\circ} \mathrm{C}$ and $700^{\circ} \mathrm{C}$ the migration of hydrogen is dominated by surface diffusion on the internal porosity, while at temperatures above $700^{\circ} \mathrm{C}$ it is controlled by "transgranular" diffusion. Above $1200^{\circ} \mathrm{C}$ the thermal dissociation rate of hydrogen is very high, so just the presence of hydrogen gas can lead to significant retention. It should be stressed that

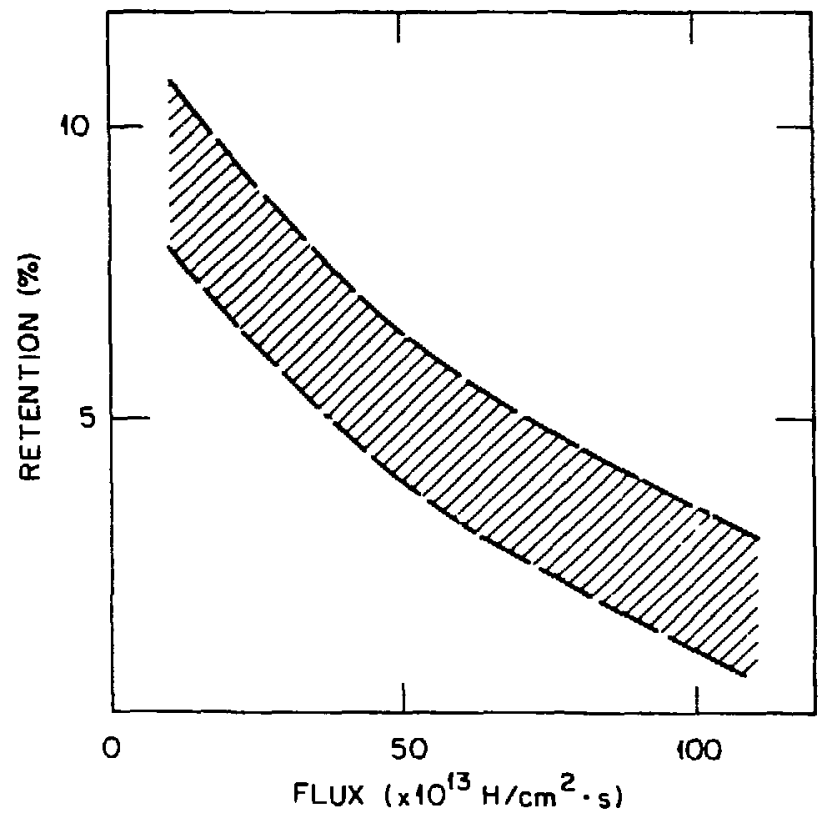

Fig. 8. Hydrogen retention as a function of incident hydrogen flux. The dashed lines indicate the upper and lower bounds of the measured values (from Ref. [22]). 
the interpretations of the results reported here depend on the existence of a significant amount of open intemal porosity. Experiments over a wider range of flux and temperature on graphites with varying amounts of porosity are needed to support these interpretations.

\section{INTRAGRANULAR}

Diffusion and trapping of hydrogen in grains appears to be significant only at temperatures above $600^{\circ} \mathrm{C}$. In addition, above $1200^{\circ} \mathrm{C}$ molecular hydrogen begins to thermally dissociate, so that the presence of hydrogen atoms no longer depends only on implanted ions and atoms. Causey et al. [24] used the DIFFUSE code to model their results at temperatures between $700^{\circ} \mathrm{C}$ and $1500^{\circ} \mathrm{C}$ for the effects of both hydrogen ions and hydrogen gas. They obtained a saturation of about $1.6 \times 10^{-5} \mathrm{H} / \mathrm{C}$ at $1200^{\circ} \mathrm{C}$. In addition, a trap energy of $4.3 \mathrm{eV}$ and a trap concentration of $1.7 \times 10^{-5}$ trap/C were obtained. In further work [26], Causey estimated the diffusivity as $D=0.93 \exp (-2.8 \mathrm{eV} / k T)$ $\mathrm{cm}^{2} / \mathrm{s}$.

\section{SPUTTERED FILMS}

Montet [4] observed that the amount of hydrogen taken up by sputtered carbon films was three to four times the amount found in the graphite cathode during a glow discharge experiment. Although no quantitative measurements of the amount of hydrogen in the film were made, it is obvious from the experimental parameters that the amount was quite large (i.e., much larger than the amount of hydrogen contained in the near-surface region). Subsequently, Hsu and Causey [25] studied the hydrogen content and thermal release of such films. They found that the properties of these films were very similar to those of the saturated near-surface layer described in Sec. III. In addition, they found that the amount of carbon sputtered was dependent on the plasma parameters and on the geometry of the experiment, especially the relative sizes of the anode, cathode, and internal components exposed to the sputtered carbon. They refer to this hydrogen-rich sputtered film as a "codeposited" film. The hydrogen content of such a film is generally near $0.4 \mathrm{H} / \mathrm{C}$ but ranges from 0.2 to $0.6 \mathrm{H} / \mathrm{C}$. Thermal desorption of hydrogen from the codeposited film is very similar to that measured for the saturated near-surface region.

In fusion experiments, these codeposited films represent a potentially large sink for hydrogen and its isotopes. Recent estimates of in-vessel tritium inventories for TFTR [27] and CГГ [28] predict that about $80 \%$ of the retained tritium will be in codeposited films. The magnitude of graphite erosion is dependent on many of the plasma parameters and, as such, depends on the device and the operational scenario.

Additional work in this area has been reported by Winter [29,30], Vietzke [31,32], Besocke et al. [33], Wilson and Hsu [34], and Causey [26].

\section{SUMMARY}

The four major processes involved in hydrogen retention in graphite have been reviewed. These processes are saturation of the near-surface region, diffusion on surfaceconnected porosity, intragranular diffusion, and erosion of carbon with concomitant formation of codeposited hydrogen-carbon films. It has been suggested that in the retention of tritium in a fusion experiment the fourth process will dominate; therefore, from that point of view, it is the most important. To address the question of plasma fuel purity, more data are needed on ion-induced release of hydrogen isotopes by hydrogen isotopes and by heavier ions (e.g., carbon and oxygen) as a function of temperature.

Much work remains to be done before accurate predictions of hydrogen rention can be made. This needed work includes a definitive experiment to determine the diffusion and retention of hydrogen on surface-connected porosity as a function of flux, fluence, and temperature; a definitive experiment to differentiate between surfaceconnected porosity diffusion and intragranular diffusion; and better definition of the erosion processes that lead to codeposited films.

\section{REFERENCES}

[1] C. A. Klein, Rev. Mod. Phys., vol. 34, p. 56, 1962.

[2] R. T. Morrison and R. N. Boyd, Organic Chemistry. 3rd ed. (Allyn \& Bacon, Boston, 1975).

[3] A. R. G. Brown and W. Watt, Industrial Carbon and Graphite, p. 86, 1958.

[4] A. G. L. Montet, in Proceedings of the 4th Conference on Carbon, 1959, p. 159.

[5] W. Möller, J. Nucl. Mater., vols. 162-164, p. 138 (1989).

[6] R. A. Langley et al., J. Nucl. Mater., vols. 76 and 77, p. $313,1978$.

[7] G. Staudenmaier et al., J. Nucl. Mater., vol. 84, p. $149,1979$.

[8] B. L. Doyle et al., J. Nucl. Mater., vols. 93 and 94, p. $551,1980$.

[9] K. Niwase et al., J. Nucl. Mater., vols. 155-157, p. $303,1988$. 
[10] K. Niwase et al., J. Nucl. Mater., vols. 162-164, p. $856,1989$.

[11] D. J. Bacon et al., j. Nucl. Mater., vols. 103-104, p. $427,1981$.

[12] J. B. Biersack and L. G. Haggmark, Nucl. Instrum. Methods, vol. 174, p. 257, 1980; D. K. Brice, private communication, 1986; W. Eckstein, IPP-9/33, MaxPlanck Institut für Plasmaphysik, Garching. 1980.

[13] M. Braun and B. Emmoth, J. Nucl. Mater., vols. 128 and 129, p. 657, 1984.

[14] D. K. Brice, Nucl. Instrum. Methods Phys. Res. B, accepted for publication, 1989.

[15] J. Roth et al., J. Nucl. Mater., vols. 93 and 94, p. 601, 1980.

[16] W. R.Wampler and S. M. Myers, J. Nucl. Maier., vols. 111 and 112, p. 616, 1982.

[17] W. Möller et al., Nucl. Instrum. Methods Phys. Res., vols. B19 and B20, p. 826 (1987).

[18] R. A. Langley et al., J. Nucl. Mater., vols. 162-164, p. $1030,1989$.

[19] R. A. Langley, J. Vac. Sci. Technol. A, vol. 7, p. 1060, 1989.

[20] H. F. Dylla et al., Nucl. Fusion, vol. 27, p. 1221 (1987)

[21] G. L. Jackson et al., J. Nucl. Mater., vols. 162-164, p. $489,1989$.
[22] R. A. Langley, J. Vac. Sci. Technol. A, vol. 5, p. 2205 , 1987.

[23] A. A. Haasz, private communication, 1989.

[24] R. A. Causey, M. I. Baskes, and K. L. Wilson, J. Vac. Sci. Technol. A, vol. 4, p. 1189, 1988.

[25] W. Hsu and R. A. Causey, J. Vac. Sci. Technol. A, vol. 5, p. $2768,1987$.

[26] R. A. Causey, J. Nucl. Mater., vols. 162-164, p. 151, 1989.

[27] A. E. Pontau et al., Fusion Eng. Des., vols. 8-10, p. 365, 1989.

[28] K. L. Wilson, Sandia National LaboratoriesLivermore, personal communication, 1989.

[29] J. Winter, J. Vac. Sci. Technol. A, vol. 5, p. 2286, 1987.

[30] J. Winter, J. Nucl. Mater., vols. 145-147, p. 131, 1987.

[31] E. Vietzke and V. Philipps, presented at the 3rd Workshop on Carbon Materials for Fusion Applications, Jülich, October 2, 1987.

[32] E. Vietzke et al., J. Nucl. Mater., vols. 145-147, p. $443,1987$.

[33] K. Besocke et al., J. Nucl. Mater., vols. 145-147, p. $651,1987$.

[34] K. L. Wilson and W. L. Hsu, J. Nucl. Mater., vols. 145-147, p. 121, 1987. 